\title{
Dictamen médico legal de un análisis de voz, ¿cómo hacerlo?
}

Medical legal opinion of a voice analysis, how to do it?

\author{
César Antonio Méndez Martínez ${ }^{1,2}$ (1) (-) \\ ${ }^{1}$ Servicio de audiología y foniatría; Hospital Dr. Luis F. Nachón; Xalapa, Veracruz; México. \\ ${ }^{2}$ Servicio de audiología y foniatría; Hospital Ángeles Xalapa; Xalapa, Veracruz; México.
}

\section{Correspondencia}

César Antonio Méndez Martínez.

E-mail: cesarfonaudio@gmail.com

\section{Gitar así}

Méndez Martínez, César Antonio. (2021). Dictamen médico legal de un análisis de voz, ¿cómo hacerlo? Revista de Investigación e Innovación en Ciencias de la Salud. 3(2), 98-118 https://doi.org/10.46634/riics.65

Recibido: 16/05/2021

Revisado: 06/11/2021

Aceptado: 23/11/2021

\section{Invited editor}

Carlos Manzano Aquiahuatl, MD, MSc.

Editor en jefe

Jorge Mauricio Cuartas Arias, Ph.D. iD

\section{Coeditor}

Fraidy-Alonso Alzate-Pamplona, MSc.

Copyright $\mathbb{C}$ 2021. Fundación Universitaria María Cano. La Revista de Investigación e Innovación en Ciencias de la Salud proporciona acceso abierto a todo su contenido bajo los términos de la licencia Creative Commons Attribution-NonCommercial-NoDerivatives 4.0 International (CC BY-NC-ND 4.0).

\section{Declaración de intereses}

$\mathrm{El}$ autor ha declarado que no hay conflicto de intereses.

\section{Disponibilidad de datos \\ Todos los datos relevantes se encuentran en el artículo. Para mayor información, comunicarse con el autor de correspondencia.}

\section{Financiamiento}

Ninguno. Esta investigación no recibió ninguna subvención específica de agencias de financiamiento en los sectores público, comercial o sin fines de lucro.

\section{Resumen}

La acústica forense es una disciplina de la criminalística que ha alcanzado una madurez analítica que obliga a que el perito en análisis de voz se especialice en adquirir conocimientos en fonética, tecnologías de sonido, habla, voz, lenguaje, patologías del habla y la voz, así como procesamiento de la señal sonora. Cuando un dictamen deba ser realizado por un profesional de la salud completamente ajeno a la técnica legal, se tropieza con una falta de protocolos, métodos y procedimientos de trabajo que le permitan entregar un informe técnico, válido y validado para la realización de una entrevista y su posterior análisis comparativo de voces, lo que promueve la necesidad de elaborar una ruta o guía metodológica a través de medios académicos físicos o electrónicos para el desarrollo de este conocimiento y su difusión profesional y científica.

\section{Palabras clave}

Dictamen médico; legal; análisis de voz; acústica forense; criminalística; voz; habla; lenguaje; patologías del habla; vocología; fonoaudiología; perito; acústica; oscilograma; voz dubitada; voz indubitada.

\section{Abstract}

Forensic acoustics is a criminalistics discipline that has reached an analytical maturity that requires the expert in voice analysis to specialize in acquiring knowledge in phonetics, sound technologies, speech, voice, language, speech, and voice pathologies, as well as sound signal processing. When an opinion must be made by a health professional completely unrelated to the legal technique, he encounters a lack of protocols, methods, and work procedures that allow him to deliver a technical, valid, and validated report for conducting an interview and its subsequent comparative analysis of voices, which promotes the need to develop a methodological route or guide through physical or electronic academic means for the development of this knowledge and its professional and scientific dissemination.

\section{Keywords}

Medical opinion; legal; voice analysis; forensic acoustics; criminalistics; voice; speaks; language; speech pathologies; vocology; speech therapy; proficient; acoustics; oscillogram; doubtful voice; undoubted voice. 
Descargo de responsabilidad El contenido de este artículo es responsabilidad exclusiva del autor y no representa una opinión oficial de sus instituciones, instituciones a las que se hagan referencia, ni de la Revista de Investigación e Innovación en Ciencias de la Salud.

\section{Introducción}

Uno de los logros del desarrollo tecnológico ha sido poder reconocer a individuos por medio de sus datos biométricos, sean estos faciales, pupilares, dactilares o de voz, generalmente a través de una muestra y la comparación con una base de datos. Para esto se han unificado criterios de la ciencia y la tecnología, por lo que puede ser habitual que se encuentren publicaciones relacionadas a este tema en diferentes campos, como la informática, el tratamiento de señales, la psicología, la física, las matemáticas, la electrónica, la lingüística, la medicina, entre otras. Sin embargo, a la par de estos desarrollos tecnológicos, la sociedad se ve enfrentada a métodos delictivos que comprometen la seguridad pública, los bienes y la vida de los ciudadanos. A pesar de que muchos de estos métodos o acciones delictivas se generan directa y físicamente sobre la víctima, se han venido presentando agresiones de manera verbal, indirectas u ocultas, que buscan generar miedo, zozobra, amenaza psicológica, difamación, discriminación, incertidumbre, por referir algunas formas, donde la única forma de identificar a dichos autores o participantes del ilícito es por el reconocimiento e identificación de su voz, convirtiéndose esta en una prueba fehaciente del accionar delictivo.

Esta técnica de identificación por medio de la voz ha sido probada y validada científicamente y tiene una gran aplicación en el ámbito legal, lo que la convierte en una actividad comúnmente ejecutada en áreas judiciales, aunque en ocasiones no se cuente con personal suficiente ni calificado para realizarla. Sin embargo, bajo mandato oficial y norma jurídica, tales áreas judiciales pueden solicitar la cooperación de personal médico potencialmente capacitado para realizar esta actividad pericial a fin de lograr el desarrollo del acto jurídico en proceso, lo que crea en el personal médico la necesidad o, mejor, el reto de buscar el escaso material bibliográfico que proporcione una guía o un protocolo de elaboración de dictámenes.

De ahí el objetivo de este artículo, de presentar unas prácticas y sencillas sugerencias para la elaboración escrita de un dictamen médico-legal de análisis de voz y, a modo de glosario, aclarar ciertos conceptos técnicos y médicos que se toman como sinónimos sin así serlo, lo que genera confusión y un dictamen impreciso, tal es el caso de los términos: "dictamen en análisis de voz", "dictamen del análisis acústico de voz", "dictamen de fonología forense", "dictamen de acústica forense", "dictamen comparativo de voz", "dictamen de foniatría forense". Cabe señalar que, aunque este escrito no se enfocará en el tratamiento de la señal o del proceso para realizar el reconocimiento comparativo entre una voz dubitada y una indubitada, se espera que este trabajo inicie la reflexión para unificar criterios y crear protocolos para un desarrollo de esta actividad que día a día comienza a tener más participación y demanda de los profesionales médicos de la voz.

\section{Dictamen médico legal de análisis de voz, ¿cómo hacerlo?}

Un profesional médico del estudio de la voz (foniatra, logopeda, fonoaudiólogo, etc.) que labora en área médica (particular o institucional), puede desarrollar cierta pericia en la identificación general de un tipo de voz e identificar algunos pacientes sin necesidad de verlos por las características propias de su voz (en caso de tener o no una patología médica específica o por la frecuencia y familiaridad de la convivencia con estos pacientes). De igual manera, en ocasiones puede identificar o descubrir 
pacientes simuladores o impostores por la voz que producen, pero complementando esta identificación al valorar el comportamiento, funciones no elocutivas y la modificación intencionada de la voz para obtener un beneficio. Esta identificación espontánea es sumamente subjetiva y sin consecuencia legal alguna.

Para presentar un contexto del accionar criminal que tiene implicaciones en este estudio, podemos indicar que en el 2020, en México, se cometieron 27.6 millones de delitos asociados a 21.2 millones de víctimas, algunas de ellas víctimas en más de dos ocasiones. Lamentablemente estas cifras no son completas porque muchas veces no se denuncian los delitos o algunos no proceden por mal planteamiento en la denuncia, por deficiencias técnicas en la correcta integración de la carpeta de investigación, deficiencias del proceso judicial o falta de recursos técnicos y humanos para el desarrollo de pruebas periciales complementarias que fortalezcan la denuncia del delito. Aquí es donde el análisis acústico de la voz como prueba pericial ha tomado fuerza y sustento como un procedimiento objetivo, no invasivo y como herramienta tecnológica, comúnmente desarrollada por personal ajeno al área de la salud.

Para ello, uno de los alcances legales de los órganos de procuración de justicia, en caso de no contar con el recurso humano capacitado para realizar esta actividad pericial, es nombrar y asignar a través del exhorto o requisición a personal que preste sus servicios en dependencias del Gobierno federal, universidades del país, asociaciones profesionales legalmente reglamentadas y reconocidas en cualquier entidad federativa de la república [1]; es decir, se pueden nombrar como peritos a profesionales de la salud con base en la Ley General del Sistema Nacional de Seguridad Pública [2]. Esta ley, en su artículo 4, faculta a la federación, al estado o al municipio para buscar la cooperación de instancias pertinentes, así como la construcción de instrumentos, políticas y servicios necesarios para el logro de sus fines, esto fundamentado en el artículo 21 de la Constitución Política de los Estados Unidos Mexicanos [3].

Un análisis de voz en el área de la salud es un procedimiento parcialmente "rutinario", que permite identificar el objetivo "forense". Acto que se vuelve -más que un reto académico- una presión profesional y personal, por los alcances legales y las potenciales repercusiones penales contra los sindicados o posibles infractores. Por lo cual saber recibir, manejar y documentar material de importancia legal, evaluar, analizar y discernir metodológicamente los resultados obtenidos, sin alterar su integridad a través de la cadena de custodia, o interactuar con individuos presuntamente relacionadas con el ilícito, así como tomar muestras o registros de voz para realizar el dictamen solicitado, es fundamental para entregar un dictamen técnico objetivo, informativo, veraz y contundente para la consideración de los órganos de procuración de justicia. Por lo anterior, la acreditación académica que tiene el personal de salud con el manejo de la voz es la de perito, quien realiza un dictamen legal.

Comúnmente se cree que el reconocimiento de voz es solo para casos de extorsión o secuestro, en cualquiera de sus modalidades, pero de acuerdo con el Código Penal de México, son muchos los delitos que pueden probarse a través del reconocimiento de la voz o documentar la planeación, relación o participación en un ilícito. A continuación se refieren una serie de delitos en que puede solicitarse la identificación de voz: delitos contra la seguridad nacional (espionaje, art. 127 / 129); delitos contra la dignidad de las personas (discriminación, art. 149); delitos contra la seguridad pública (asociación delictuosa, art. 164); delitos en materia de vías de comunicación (art. 166 / 168); delitos contra la autoridad (art. 178 bis, 179, 180 bis); delitos contra la salud (actos en materia de narcóticos, art. 193 / 199); peligro de contagio (art. 199 bis); delitos contra los derechos reproductivos (art. 199 ter-sextus); delitos 
contra la indemnidad de privacidad de la información sexual (art. 199 septies); delitos contra el libre desarrollo de la personalidad (corrupción de menores de 18 años, pornografía, turismo sexual, lenocinio, trata de personas, pederastia, art. 200 / 209 ter); revelación de secretos y acceso ilícito a sistemas y equipos de informática (art. 210, art. 211 bis); delitos por hechos de corrupción (art. 212) ejercicio ilícito de ejercicio público (art. 214) abuso de autoridad (art. 215) desaparición forzada de personas (art. 215), coalición de servidores públicos (art. 216); uso ilícito de atribuciones y facultades (art. 217); del pago y recibo indebido de remuneraciones de los servidores públicos (art. 217 ter-quáter); concusión (art. 218); intimidación (art. 219); ejercicio abusivo de funciones (art. 220); tráfico de influencias (art. 221); cohecho (art. 222); peculado (art. 223); enriquecimiento ilícito (art. 224); responsabilidad profesional (disposiciones generales, art. 228-230; delitos de abogados, patronos y litigantes, art. 231-233); falsedad (falsificación de documentos, art. 243-246, falsedad en declaraciones judiciales e informes dados a una autoridad, art. 247, 248; variación del nombre o del domicilio, art. 249, usurpación de funciones públicas o de profesión y uso indebido de condecoraciones, uniformes, grados jerárquicos, divisas, insignias y siglas, art. 250- 251 bis); delitos contra la libertad y el normal desarrollo psicosexual (hostigamiento sexual, abuso sexual, estupro y violación, art. 259 bis - 266 bis, incesto, art. 272); delitos contra el estado civil y bigamia (art. 277 - 279); delitos contra la paz y seguridad de las personas (amenazas y cobranza extrajudicial ilegal, art. 282 - 284 bis); allanamiento de morada, art. 285 - 287); delitos contra la vida y la integridad corporal ( feminicidio, art. 325 - 328, aborto, art. 329 - 334, abandono de personas, art. 335 - 343, violencia familiar, art. 343 bis- 343 quáter); privación ilegal de la libertad y otras garantías (art. 364, 366 ter-quáter); delitos en las personas contra su patrimonio (robo, art. 367, 368, 372, 373, 376, 378, 381, abuso de confianza, art. 382-385; fraude, art. 386 - 389; extorsión, art. 390; fraude familiar, art. 390 bis; despojo de cosas inmuebles o de aguas, art. 395, 396; daño en propiedad ajena, art. 397); encubrimiento y operaciones con recursos de procedencia ilícita (art. 400); delitos electorales y en materia de registro nacional de ciudadanos (art. 401 -413); delitos contra el ambiente y la gestión ambiental (de la bioseguridad, art 420 ter); de los delitos en materia de derechos de autor (art. 424-427) [1].

Hay que hacer una aclaración importante; de los ejemplos anteriormente citados no son únicos o aislados, pueden ser combinados y no en todos se solicita la intervención del perito en análisis de voz, solo en aquellos en los que la esencia del delito requiera el análisis e identificación de la voz y a consideración de la autoridad competente, pudiendo ser solicitado la intervención durante la averiguación previa, proceso de instrucción o juicio para documentar la participación y el grado de implicación en los hechos. La realización de un peritaje, al igual que en la medicina forense, no determina responsabilidad ni señala directamente penalidades, sino que aporta pruebas o evidencias técnicas y estudios científicos para que los operadores judiciales (jueces y magistrados) impartan justicia [4].

Aunado a lo anterior, se puede decir que no basta tener solo conocimientos médicos foniátricos para realizar los análisis periciales, sino que es necesario conocer y aplicar principios básicos de otras materias a fin de soportar y dar fortaleza al dictamen. Por desgracia no existe una guía de sugerencia para la realización de este tipo de periciales. En la literatura médica y algunas referencias o artículos encontrados, solo se menciona de manera general el propósito del peritaje mismo, su alcance o potenciales conclusiones, quizás referido al perfil de preparación profesional, académica o de experiencia del perito. Algunas de estas publicaciones se encuentran en áreas afines como criminalística, derecho, filosofia, informática, lingüística, psicología, etc. [4]. 


\section{Reconocimiento acústico de la voz. Antecedentes}

Como referencia histórica y en alusión al interés que se ha desarrollado para poder identificar una voz con fines legales y sin confundir con estudios en desarrollo del reconocimiento de voz biométrico para mandos, en 1947, Potter Kopp y Green publicaron el libro Visible speech, a través del cual pretendían instruir sobre la interpretación lingüística del habla, ya que habían codificado el habla en formas gráficas (espectrogramas o sonogramas), utilizando una máquina de reciente invención: el espectrógrafo analógico de sonido o sonógrafo, el cual permitía la representación del sonido hablado en una referencia tridimensional (frecuencia/amplitud/tiempo) mediante la realización de sucesivos análisis de Fourier a corto plazo en una muestra de voz [5].

En los años 60, con la denominación de voiceprint, Lawrence G. Kersta, de laboratorios Bell, con un espectrógrafo inició ensayos para la identificación del habla y música relacionados con sistemas de comunicación, que le permitió obtener un registro energético y frecuencia temporal denominado por él como sonograma, que permitió concluir que la voz humana es única y puede dejar una "huella" acústica, considerándola similar a la dactilar llamando a esta analogía voiceprint identification/fingerprint identification. Prácticamente siendo este el primer peritaje en análisis de voz [6].

En ese tiempo, Kersa también sostenía que la individualidad de la voz se fundamenta en el mecanismo fisiológico de la producción de voz y el aprendizaje para el proceso del habla de cada persona, donde las cuerdas vocales y los resonadores fisiológicos son los que dan individualidad (características anatómicas que son irrepetibles en cada humano), al igual que los elementos de movilidad dinámica individual (labios, dientes, lengua, paladar blando, músculos masticadores) modifican particularmente la emisión de sonidos de la persona que los originaba, independientemente de quién o qué estuviera presente o junto; ya que esto último podía ser un evento que podía modificarse; tomando en cuenta al estado de salud, comportamiento simulación o intención y de esta forma poder variar el resultado o interferir en la correcta identificación del individuo.

En 1979, Oscar I. Tosi, uno de los principales padres del voiceprint, y a petición del FBI, evaluó dicha técnica desde aspectos científicos, técnicos y jurídicos, y determinó en el artículo $O n$ the theory and practice of voice identification, que la identificación de la voz hasta ese momento era un estado imperfecto, tanto jurídica como técnica, ya que se contaminaba con hallazgos de susceptibilidad al control de cambio voluntario (mímica y disfraz), y presentaba una notable falta de fundamentos sólidos en operación, métodos de aprendizaje del proceso, falta de principios de validez metodológica y aseveraciones que influían en el estado de exactitud de las conclusiones, lo que generaba más confusiones y controversias que resultados contundentes [7].

Esta breve referencia histórica del análisis de voz, en que se detecta y resalta que el proceso de información del locutor está completamente imbricado con el resto de la información de la señal de la voz, permite inferir que la realización e integridad de los componentes pueden modificarse de manera intencionada o súbita y ser sumamente perjudiciales para la correcta identificación de la voz, esto debido a que es un proceso dinámico expuesto a múltiples modificaciones (órganos de producción, variables modificadas, propagación sonora hasta el oído receptor o fuente de registro, características individuales de la fuente de registro como son amplificadores, filtros, canales, sistema de almacenaje digital o analógico, compresiones, codificaciones, resolución, etc.). 
En la actualidad la sección de tecnología y vigilancia electrónica del FBI es el área responsable de la identificación de voz y para ello trabaja básicamente con el método auditivo-espectrográfico, como norma para no acudir a testificar a los tribunales y utilizar dicho método solamente como apoyo a la investigación de sus propios casos o de aquellos en los que son requeridos por otros organismos policiales o fuerzas de seguridad. Ya en el ámbito privado, la mayoría utiliza el método espectrográfico, avalado por el subcomité de Análisis Acústico e Identificación de Voz (VIAAS, por sus siglas en inglés) de la International Association for Identification (IAI). Y a nivel internacional por la International Association for Forensic Phonetics and Acoustics (IAFPA, por sus siglas en inglés), organización que busca fomentar la investigación y la creación de un foro de intercambio de ideas e información sobre la práctica, desarrollo e investigación sobre fonética y acústica forense, para establecer y hacer cumplir los estándares de conducta así como procedimientos profesionales para aquellos involucrados en el trabajo de casos fonéticos y acústico forenses. Dicha organización se instaló en York, Inglaterra, en 1991, siendo la más activa de Europa [8]. Asimismo, cabe destacar las siguientes instituciones: el Instituto Nacional de Investigación de Ciencia Policial de Japón, el Laboratorio de Análisis y Tratamiento de la Señal de la Policía Técnica y Científica de Francia, el Laboratorio de Acústica Forense de la Comisaría General de la Policía Científica de España, así como la gran mayoría del resto de los laboratorios policiales o laboratorios forenses dedicados a la identificación de hablantes pertenecen a la IAI y diversos cuerpos policiales o de investigación gubernamental asociados a la IAFP.

Finalmente, y respecto a América, cabe citar universidades como la Escuela de Medicina Paulista de Sao Paulo, el Departamento de Medicina Legal de la Universidad de Campinas en Brasil, la Universidad de Buenos Aires, la Universidad de Perú, el Departamento Administrativo de Seguridad y Policía Nacional de Colombia, el LACRIM (Laboratorio de Criminalística de la Universidad Austral de Chile), el INACIPE (Instituto Nacional de Ciencias Penales de México) el Departamento de Lingüística Forense de la UNAM, la Fiscalía General de la República de México, así como otras instituciones en diversos países de Latinoamérica (Argentina, Paraguay, Perú, Colombia, Brasil, Honduras [5].

Por lo que se reafirma en términos generales no basta tener los conocimientos básicos de medicina (anatomía y fisiología del aparato fonoarticulador) o de foniatría (estudio de la voz) sino también es necesario tener algunos conocimientos o conceptos básicos de lingüística (estructuración de discurso, lexicología, regionalismos, modismos, sintaxis, semántica, morfología lexicológica, procesos psicopatológicos del habla, etc.), fonética articulatoria, acústica, criminología, criminalística, sociología (rasgos psicosociales, entorno social, demográfico, etc.), informática, metodología y derecho; solo por citar unos ejemplos; de los conocimientos básicos que nos podrán ayudar a individualizar las características propias del dictamen a realizar [9].

Si bien todo depende de la forma individual de trabajar y realizar un dictamen hay que recordar que existen diferentes técnicas de análisis, siendo la más representativas:

- Análisis fonético auditivo.

- Análisis fonético acústico.

- Análisis fonético acústico con análisis fonético auditivo.

- Análisis por reconocimiento automático de voces.

- Sistema de análisis de reconocimiento automático del locutor. 
- Sistema de análisis de reconocimiento del locutor por analista humano.

- Análisis fonético espectrográfico.

- Análisis bajo complemento de redes neuronales, etc. [10].

En la formulación del peritaje debe considerarse, además, la emisión de valoraciones en forma individual o conjunta, por proceso colegiado, mediante combinación de puntuaciones por escala clásica de probabilidades, o por índice de probabilidad, utilizando metodologías basadas en valoración de verosimilitud por mezclas gaussianas, modelos ocultos de Markov, cuantización vectorial o redes neuronales entre otras [5].

Por todo lo anteriormente comentado, a continuación se describen los puntos a seguir como sugerencia para la realización de un dictamen pericial en análisis de voz desde el punto de vista del área de la salud a reserva de las consideraciones propias de elementos a disposición de cada caso en particular y de lo solicitado de manera específica:

\section{Identificación general}

La labor del perito debe basarse en la independencia y el rigor científico. Aunque pareciera trivial y rutinario siempre hay que indicar fecha y lugar de realización de nuestro dictamen; seguido por la identificación alfanumérica propia del dictamen que se realiza, identificación propia del proceso en acción (carpeta de investigación, proceso penal, expediente o denominación particular); agregar el motivo o "asunto" a tratar; es decir, referencia específica al tipo de dictamen solicitado (dictamen en la especialidad de análisis de voz, dictamen en materia de fonología forense para identificar, dictamen para realización de pericial forense en análisis de voz, dictamen comparativo de reconocimiento de voz, dictamen de fonología forense para identificación de individuo, dictamen forense de acústica para identificación de fenómenos agregados - análisis del ambiente acústico--, dictaminación de análisis de voz y reporte estenográfico de grabaciones; etc. ); persona a quien se envía en respuesta a la solicitud inicial o a quien va dirigida (generalmente persona con investidura legal quien solicitó la realización de dicho pericial). Referencia que se puede ver en el Esquema 1.

Ciudad de México; 17 de septiembre del 2021

FOLIO: 01234

CARPETA DE INVESTIGACION: 031/2021-2

PROCESO PENAL CI/UECS/MX/0321EXPEDIENTE

ASUNTO: Dictamen en la especialidad de análisis de voz

C. LIC. A.M.G. (NOMBRE)

DIRECCION DE LA UNIDAD ESPECIALIZADA EN COMBATE AL SECUESTRO

FISCALIA GENERAL DE LA REPUBLICA

PRESENTE

Esquema 1. Identificación general e inicial del dictamen a realizar. 


\title{
Antecedentes de asignación, requisición o designación
}

Aunque parezca repetitivo, cabe nuevamente mencionar la solicitud que fundamenta nuestra intervención y el tipo de dictamen a realizar, haciendo referencia del oficio de comisión o aceptación de cargo, donde se especifique el lugar y fecha en que fue recibido y si fue dirigido de manera directa o a través de la Junta de Gobierno de la Unidad Hospitalaria o del director del hospital; o de cualquier organismo o estamento; así como especificar el término legal establecido para la realización de dicho pericial; y salvo que sea absolutamente necesario referir en qué condiciones será realizado, véase ejemplo en Esquema 2.

... En atención a la petición realizada mediante oficio identificado como FGR/DUECS/01234/2021, emitido el día 17/09/2021 y recibido por el abajo firmante el día 21/09/2021 en "X" (lugar); en que se solicita al director de la unidad Hospitalaria "Hospital México" la designación del abajo firmante para intervenir como perito en materia de Análisis de voz, relacionado con la carpeta de investigación $\mathrm{Cl} /$ UECS/MEX/2021; en un término no mayor a 10 (DIEZ) días hábiles, en el CEFERESO (Centro Federal de Readaptación Social México) ...

Esquema 2. Párrafo correspondiente a justificación de la designación a realizar dictamen.

\section{Planteamiento del problema ("a efecto de lograr...")}

De manera concreta, se circunscribe a especificar claramente las interrogantes establecidas y provenientes de los hechos, así como los fenómenos o circunstancias observadas, al aplicar principios de criminalística, por lo que el perito deberá plantear de manera específica la relevancia de cada uno de ellos mediante técnicas de observación, exploración, descripción y correlación para explicar los fenómenos o causas que conlleven a aclarar, confirmar o desechar un objetivo específico [9], es decir, ser un auxiliar del derecho penal que se ocupa del descubrimiento y verificación científica del delito y del delincuente. Véase ejemplo en Esquema 3.

\begin{abstract}
... Realizar dictamen de análisis de voz y su pericial correspondiente a los \# audios contenidos en (elementos de almacenaje de información proporcionados) mismos que pueden ser considerados como dubitables y a la muestra de voz de los imputados U.P.I. y B.O.L.I. (mismas que deberán de tomarse como indubitables) y de esta manera debiendo determinar si corresponde o es la misma que obra en la muestra de voz de los vinculados a proceso...
\end{abstract}

Esquema 3. Párrafo correspondiente a mostrar una sugerencia de planteamiento del problema a peritar.

\section{Descripción de los elementos de estudio}

Debe hacerse una descripción amplia y explícita de todos y cada uno de los elementos proporcionados para analizar, detallar o limitar el entorno de trabajo (número y tipo de elementos proporcionados y qué tipo de archivos pueden llegar a contener, tipo de archivos contenidos dentro de cada uno de ellos, características de identificación originales y agregadas, estado de integridad o conservación del embalaje original o del utilizado últimamente para su transporte y entrega, seguido por su procedimiento de apertura secuencial a fin de identificar su integridad y conservación, o situaciones especiales en que además de recibir los elementos a estudiar se proporcionen también anexos impresos para complementar su integridad y de 
ser necesarios comparativos para su análisis todos ellos identificados de manera global e individual a fin de conservar dicha integridad y características de embalado y etiquetado para correspondencia de su integridad en la cadena de custodia (ver Esquema 4).

... 1) Se recibe 1 (UNO) disco compacto "CD" marca SONY número identificativo de serie G6230KAA70161806 rotulado caligráficamente con la leyenda "GRABACIONES" mismo que se encuentra dentro de una caja plástica propia de empaque.

2) Se recibe 1 (UNO) disco compacto "CD" marca SONY número identificativo de serie K2472QAG9037342 rotulado con etiqueta blanca adherible rotulada caligráficamente con la leyenda "10 grabaciones de celular", mismo que se encuentra dentro de sobre blanco de papel sin rotular parcialmente roto.

3) se recibe memoria "USB" Kingston azul agua con cubierta metálica girable, misma que se encuentra dentro de una bolsa transparente nylon y que contiene etiqueta adhesiva verde fosforescente con la leyenda "entrevista"

4) teléfono celular negro impresión de fábrica en su parte posterior "LG", apagado, con cargador de energía para conexión a pared color negro dentro de bolsa nylon transparente y con etiqueta adhesiva blanca rotulado caligráficamente como "2 conversaciones"

5) continuar descripción de cada uno y de todos los elementos proporcionados.

Esquema 4. Párrafo referente a la descripción de los elementos a analizar.

\section{Metodología y técnicas aplicadas para realizar el dictamen}

Considerando que la elaboración de un dictamen pericial no puede ser repetitivo, ya que cada uno presenta variabilidad de elementos, no siendo factible a ser analizados de igual manera con base en la experiencia, es necesario plantear una metodología acorde y específica para obtener un fin, siendo el método científico la guía sistemática para la aplicación secuencial de pasos a desarrollar para entender o comprender el problema previamente planteado.

De este modo el diseño para la recolección de datos, formulación de interrogantes, análisis teórico y sistemático, previamente comentados en el apartado de "planteamiento del problema"; permitirá tener una conclusión de resultados potencialmente reproducibles considerando su variabilidad y repercusión de la certeza científica y por ende legal.

El método deductivo, en esencia, señala el camino para conocer, de varios principios universales, una verosimilitud particular, con el objeto de verificar si el hecho concreto que se investiga se enmarca o se tipifica en los fenómenos producidos y observados. Por lo que la actividad científica se lleva a cabo sometiendo los principios universales a la forma lógica más común del razonamiento deductivo, mediante el manejo de varias premisas o proposiciones lógicas del silogismo, cuya conclusión verdadera deducimos de la primera por medio de la segunda [11]. Por lo que se procede con:

\section{Valoración cualitativa}

Descripción individual de cada uno de los elementos a estudiar, detallando su ubicación (contenedor), descripción de su orden de aparición de acuerdo con la apertura electrónica o reproducción; observación e identificación general de cada uno de los archivos describiendo la percepción auditiva detectada (conversación, monólogo, elementos sonoros comunes potencialmente reconocibles, ruido de fondo, nivel de inteligibilidad ininteligibilidad, ruido, espacios de silencio, etc.); así como su potencial utilidad auditiva, además de especificar y detallar qué archivos son considerados para analizar, especificando de ser así las condiciones que los hacen potencialmente deficientes para su análisis (descripción en el Esquema 5). 


\begin{tabular}{c|c|c}
$\begin{array}{c}\text { Nombre del } \\
\text { archivo }\end{array}$ & \multicolumn{1}{|c|}{ Observaciones (percepción auditiva general) } & Utilidad \\
\hline 160701_010 MP3 & $\begin{array}{l}\text { Se aprecia conversación, saturación por ruido de fondo distorsión, } \\
\text { tiempo, tamaño. }\end{array}$ & Sí \\
\hline 160701_011 MP3 & Se aprecia conversación (dos personas) tiempo, tamaño. & Sí \\
\hline 160701_015 MP3 & $\begin{array}{l}\text { Se aprecian voces múltiples, ruido de fondo, saturación, distor- } \\
\text { sión, zumbido constante, periodo ininteligibilidad, tiempo, tamaño }\end{array}$ & Con reservas \\
\hline 160701_016 MP3 & Se aprecia múltiples sonidos ambientales, saturación & No
\end{tabular}

Esquema 5. Descripción cualitativa de los elementos analizados.

\section{Valoración cuantitativa}

Descripción individual para obtener una totalidad en general de cada uno de los elementos de contención y tener una cantidad total general de archivos a estudiar. Se incluyen aquellos que por alguna situación no hayan sido utilizados y se debe detallar por qué no son fiables o aptos al análisis (deteriorado, contención, etc.). Véase ejemplo descriptivo en el Esquema 6.

Disco compacto “CD” marca SONY número identificativo de serie K2472QAG9037342

\begin{tabular}{c|c|c|c|c|c}
\hline Num & Nombre del archivo & Voces de interés & Longitud & SNR & Consideración para estudio \\
\hline 1 & 160704_0012 mp3 & H 01_voz & $38.4 \mathrm{Seg}$. & $30.0 \mathrm{dBs}$ & Test reproducción a reserva \\
\hline 2 & 160704_0014 mp3 & H 04_voz & $11.6 \mathrm{seg}$. & $26.87 \mathrm{dBs}$ & Test comparación probable \\
\hline 3 & 160704_0022 mp3 & F 02_voz & $19.2 \mathrm{seg}$. & $29.56 \mathrm{dBs}$ & Modelo comparativo probable
\end{tabular}

Esquema 6. Descripción cuantitativa de los elementos analizados.

\section{Proceso de la toma de muestras y análisis de archivos}

Dado a que el presente artículo presenta unas sugerencias para realizar el dictamen pericial de análisis de voz de manera escrita o como redactarlo en una forma resumida y sencilla; a continuación, y a modo de representatividad del proceso, se referirá a tres estrategias fundamentales y básicas para el reconocimiento comparativo de la voz y a los mecanismos o procedimientos en los que se basan las interfaces de reconocimiento de voz debido a que son varias y muy variables para cada institución o persona. La primera se basa en la utilización de técnicas de comparación de patrones, la segunda en la obtención de características fonéticas y la tercera en realizar una mezcla de las dos anteriores.

Se insiste en que hay varias estrategias para abordar el reconocimiento de voz. Y cada perito las aplica o utiliza de manera particular, de acuerdo con su experiencia y comodidad, con los elementos proporcionados, los medios de reproducción así como del equipo disponible y lo requerido por la autoridad. Se recuerda que para estos procesos hay una diversa bibliografía para al análisis de la señal sonora de la voz [12]. 
Teniendo en cuenta lo anterior, y de manera general, todas las muestras de sonido obtenidas a través de cualquier dispositivo electrónico se encuentran codificadas en el dominio del tiempo; por lo tanto, pasar del dominio del tiempo al de la frecuencia en un intervalo considerado (ventana) proporcionará un conjunto de patrones en este nuevo dominio, limitado por el ancho de banda de la señal. Todo esto a través de la transformada de Fourier. ejemplo que puede verse en la Figura 1 [13]

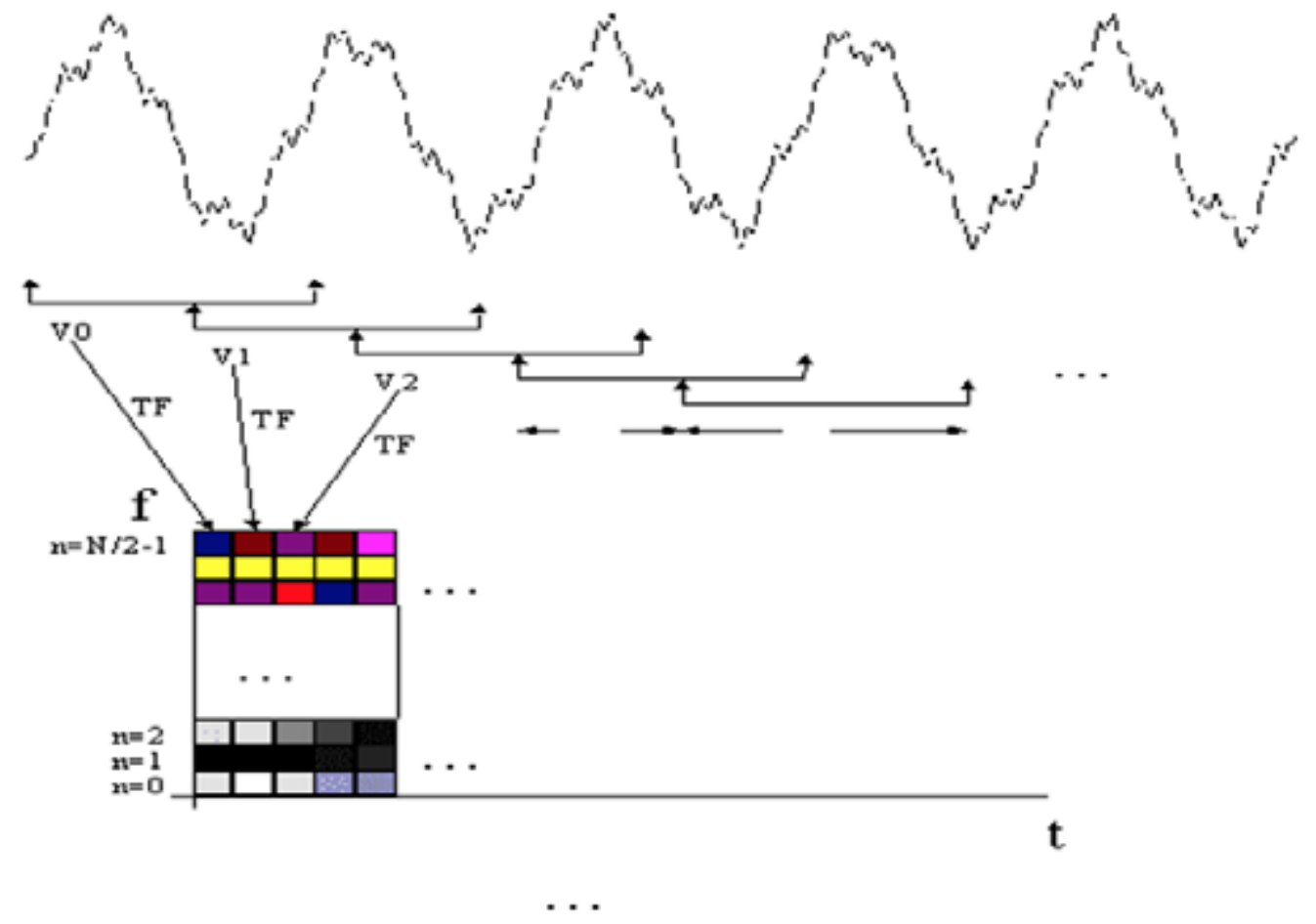

Figura 1. Traspaso de la señal acústica del dominio temporal al dominio de la frecuencia.

Por lo que respecta a la técnica de comparación de patrones, esta estrategia tiene su principal ventaja en que no necesita descubrir características espectrales de la voz, aunque su principal dificultad está en tener una base de datos de patrones similares significativa, además de carecer del significado fonético directo; sin olvidar que la técnica de comparación de patrones tiene que partir de una representación acústica tiempo-frecuencia (ver Figura 2) [13].

Esta estrategia basa su funcionamiento en el establecimiento de una distancia entre vectores, de tal manera que se puede calcular la distancia de cada patrón proveniente de las muestras de voz con respecto a la base de datos existente (proporcionada para análisis).

La base de datos de patrones se obtiene seleccionando grupos significativos de palabras o frases que se pretenden reconocer, estos grupos pueden estar constituidos por unidades tales como sonidos básicos (fonemas y alófonos) difonemas, demialofonos, palabras, frases, etc. Una vez seleccionado el grupo básico se realiza la grabación de estos sonidos (con un contexto asociado a los archivos en revisión) y se procede a obtener sus características espectrales, habitualmente parámetros de Linear Predictive Coding (LPC) o coeficientes mel-cepstrales, esquemáticamente representados en la Figura 3 [13]. 
Dictamen médico legal de un análisis de voz

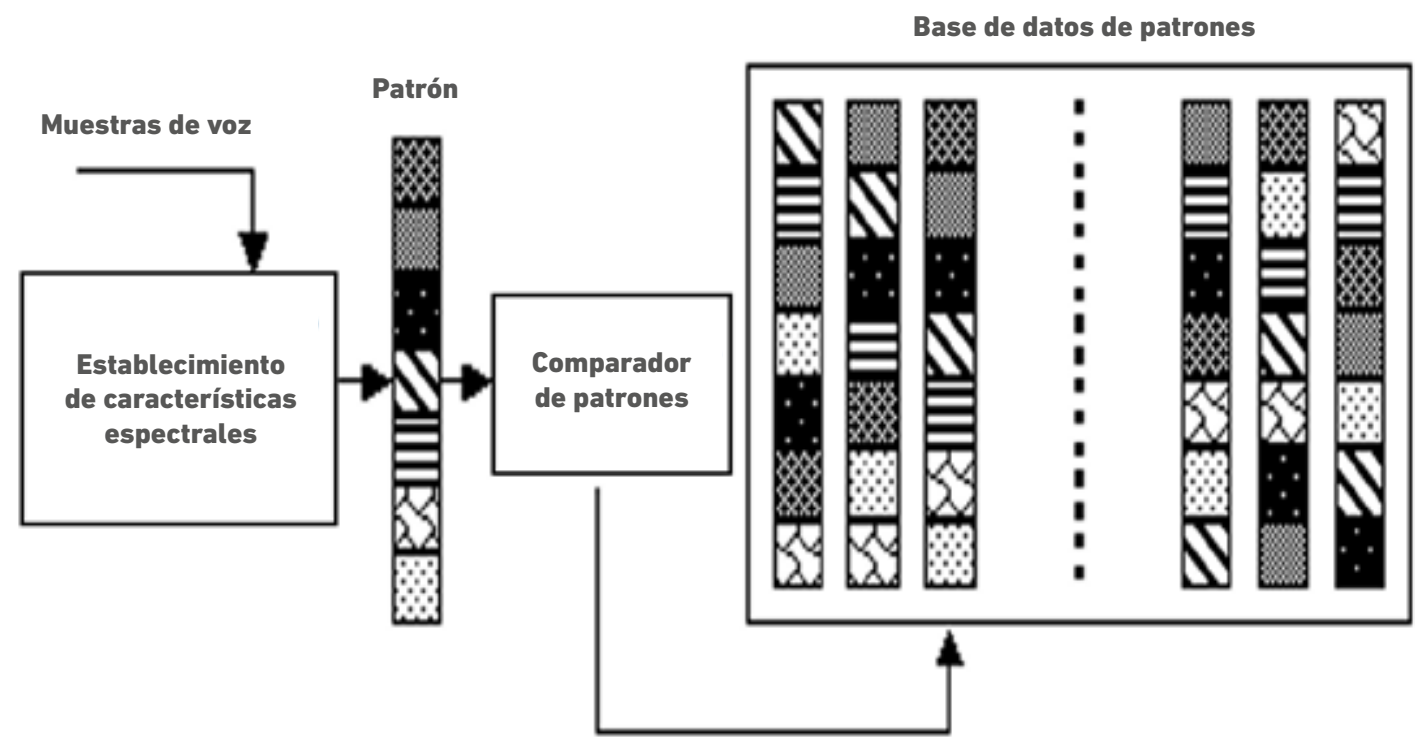

Distancia mínima

Figura 2. Comparación de patrones con la base de datos.

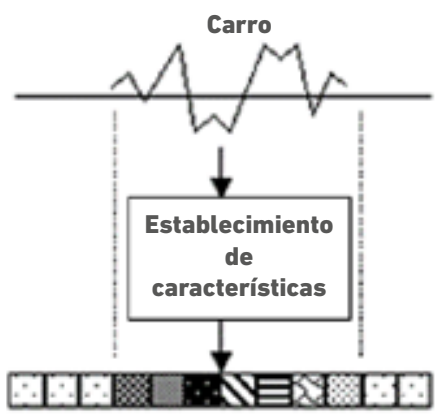

Base de datos de patrones
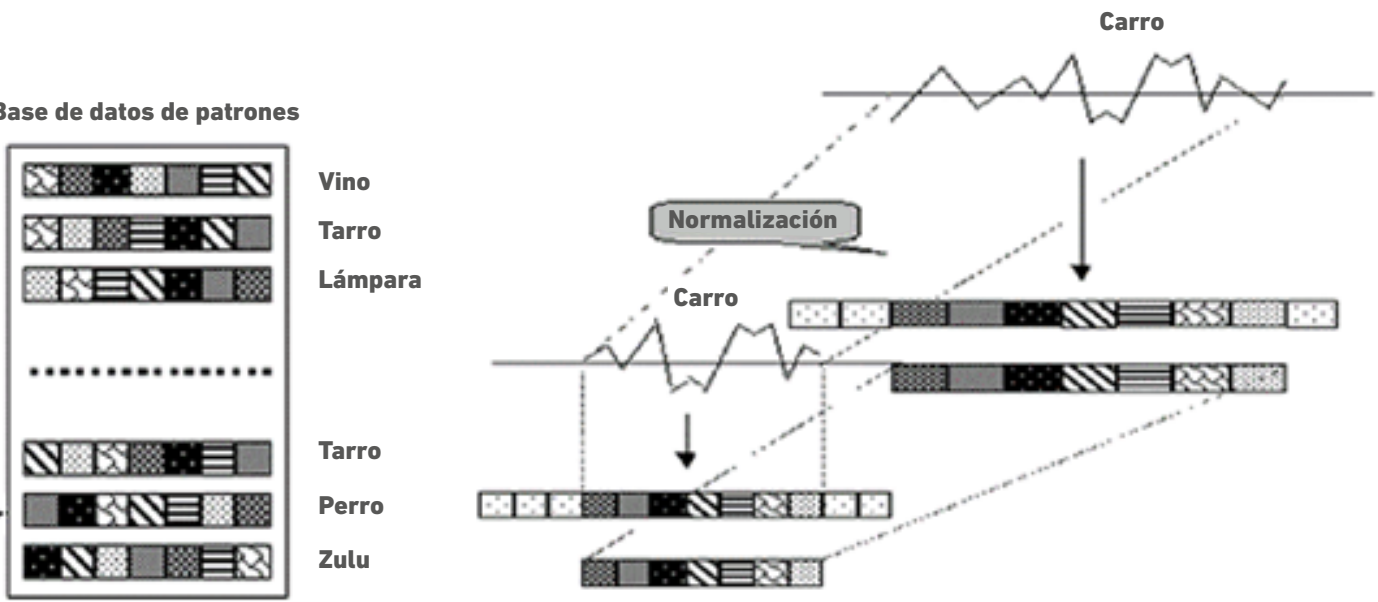

Figura 3. Selección significativa y específica de una palabra /carro/ 
Una vez que se obtiene una muestra de voz, misma que previamente se ha considerado como potencialmente reproducida y comparada con los archivos de sonido recibidos para su análisis (es de resaltar que no se conoce exactamente su origen, modo, lugar y sistema de registro y ha sido generada por "extensión" controlada / palabra, frase o texto/ en un ambiente potencialmente distinto al original), se procede a hacer una revisión comparativa con los patrones seleccionados y así poder detectar una coincidencia o compatibilidad entre ambas muestras o patrones y determinar el grado de diferencia o similitud. Lo que dará la potencial certeza a dictaminar si esta evidencia dubitada (voz grabada de la que existen dudas en torno a la identidad de la persona que habla) corresponde o no con la evidencia indubitada (grabación en la que no existen dudas respecto a la identificación de la persona que habla) [14] obtenida y cotejada. De manera general se representa en los Esquemas 7a y 7b.

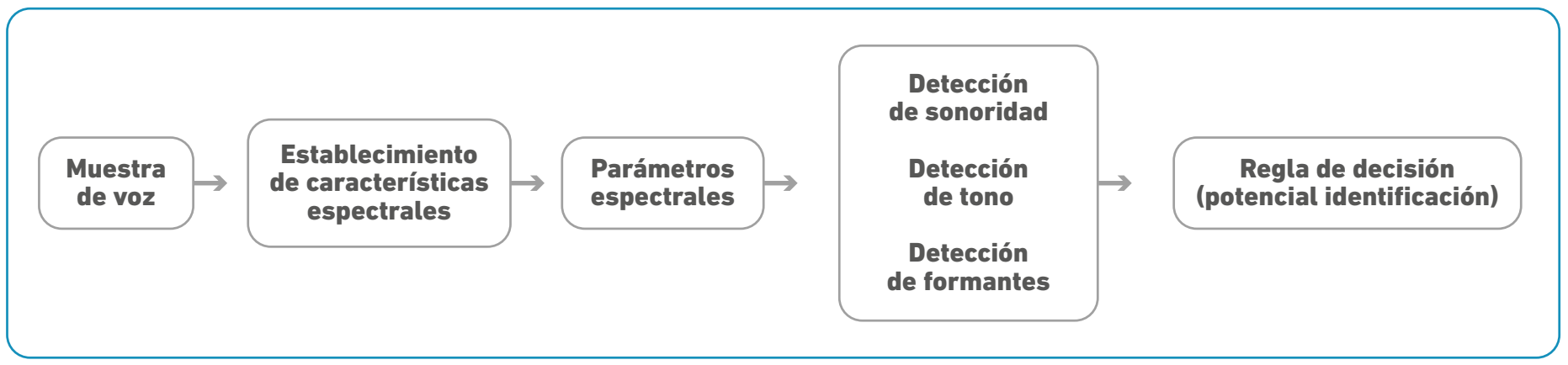

Esquema 7a. Selección de patrones.

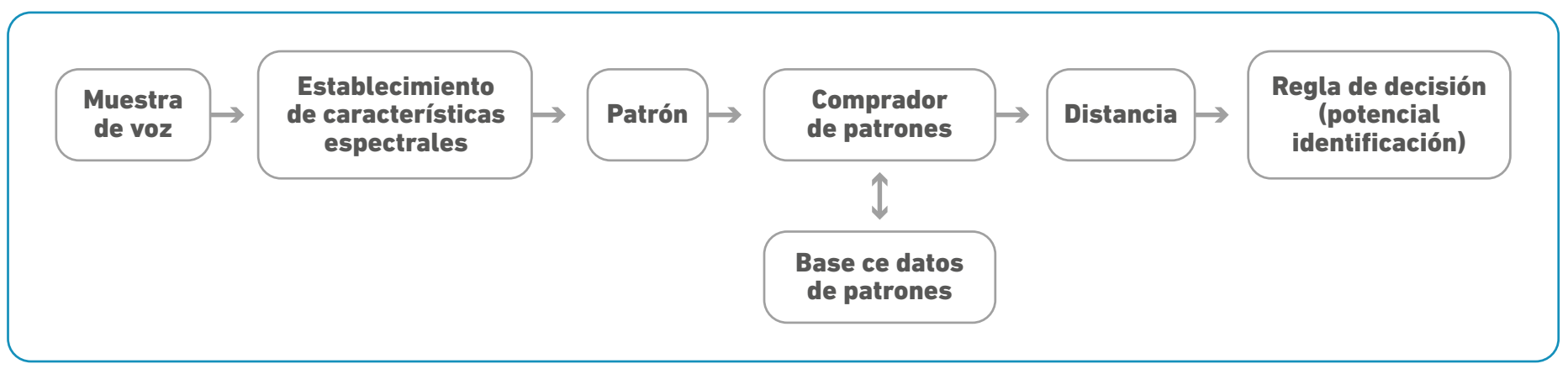

Esquema 7b. Comparación del patrón.

\section{Análisis espectrográfico}

Dicho análisis se realiza con el fin de observar las características específicas de algunos fonemas y de la pauta potencial de correspondencia e información contenida por segmentos de voz e interés detallado a la observación de armónicos formantes y ruido de la señal [15] para confirmación o descarte de utilidad. Misma que se puede observar en las Figuras 4, 5a y 5b). 

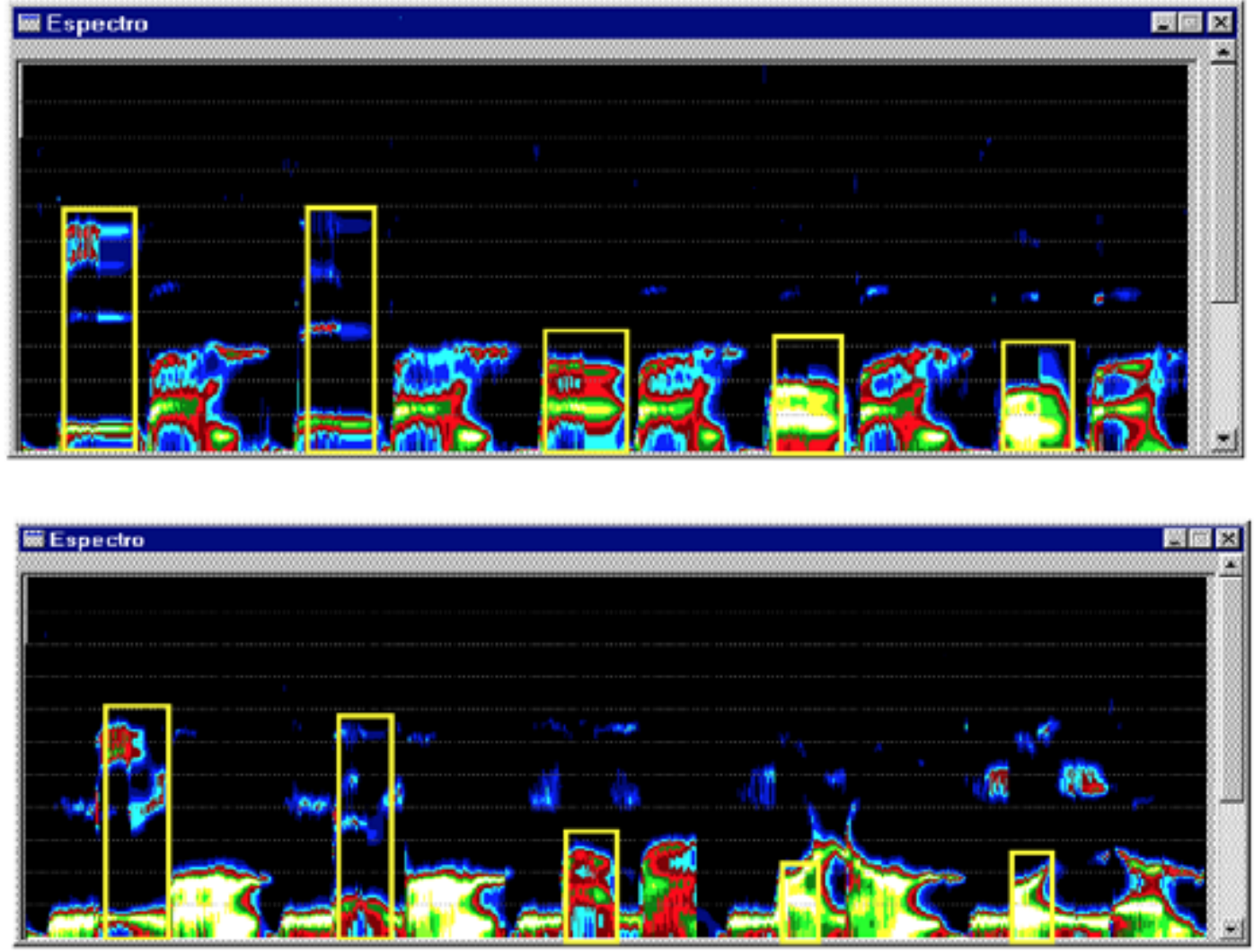

Figura 4. Espectrograma de sonidos vocálicos: /pipa, pepa, papa, popa, pupa/ Cuadro superior. /mimo, memo, mama, mono, mundo/ Cuadro inferior.

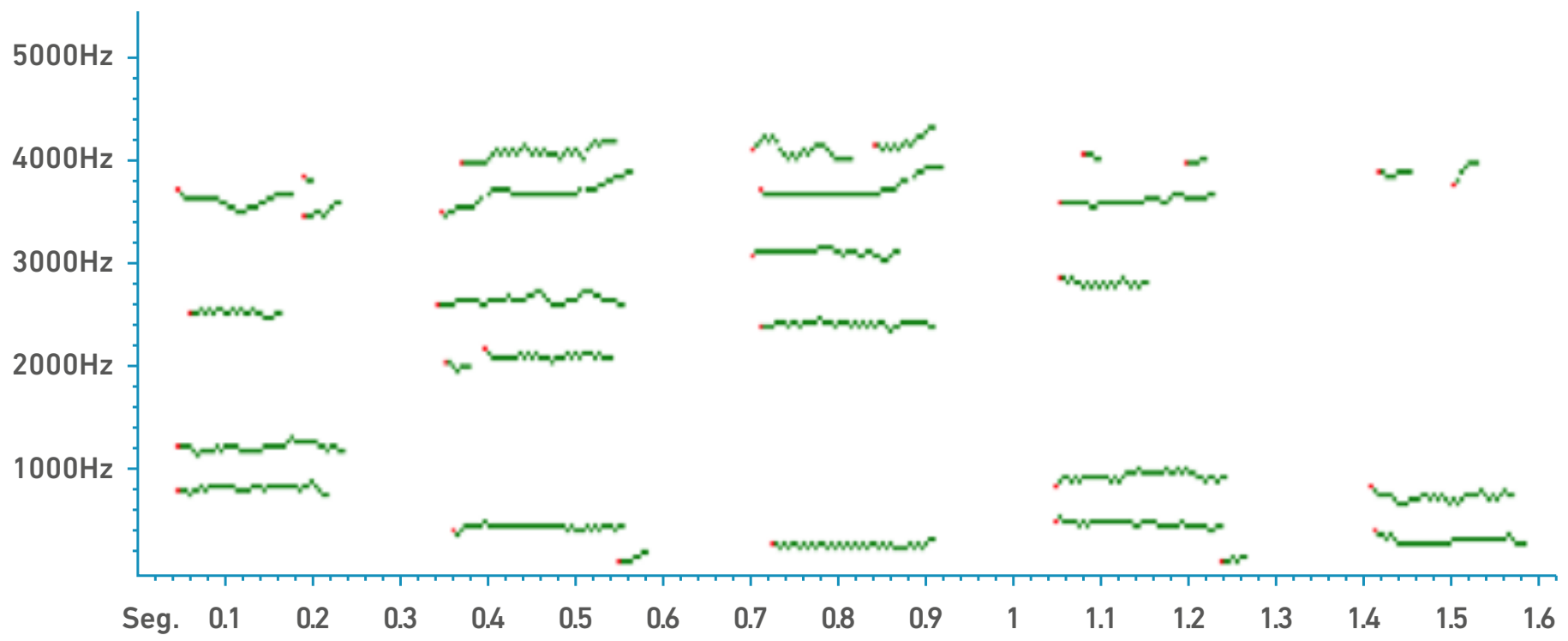

Figura 5a. Espectro de la secuencia "a, e, i, o, u" formantes. 


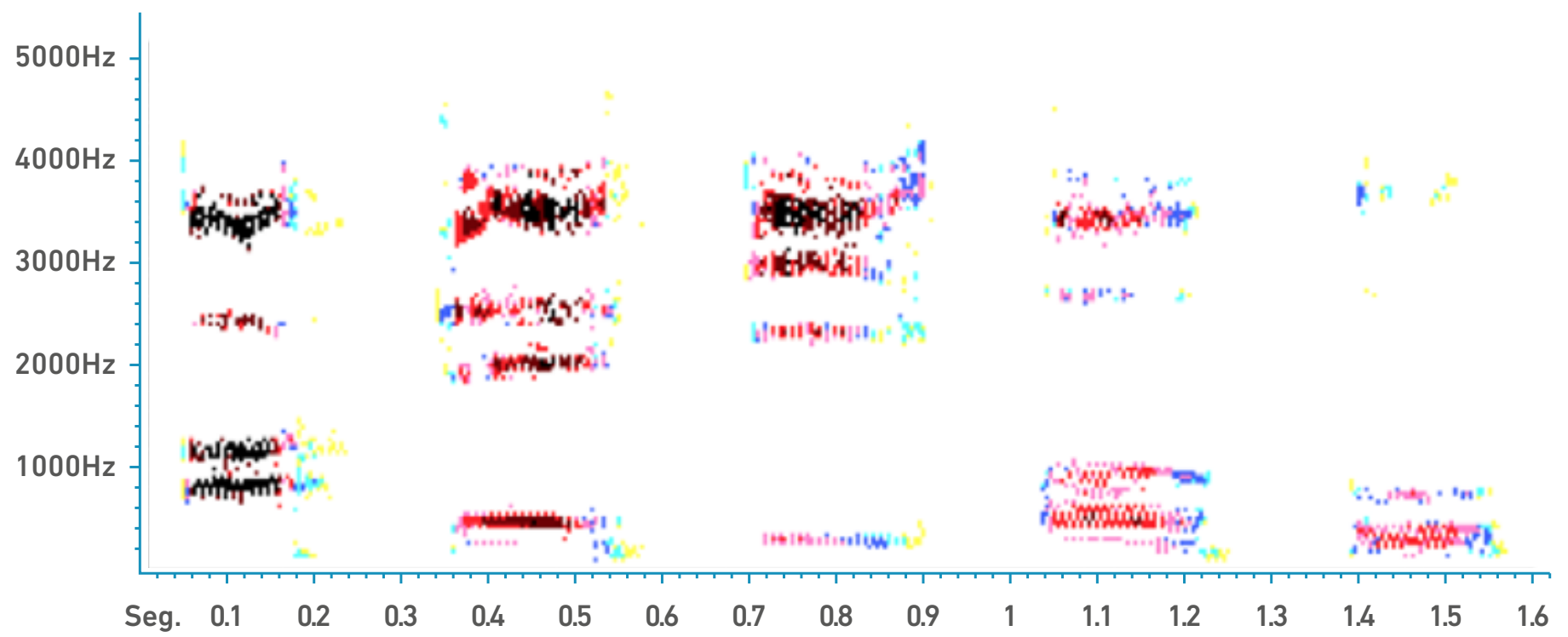

Figura 5b. Espectro de la secuencia “a, e, i, o, u” formantes.

Si bien estos ejemplos de análisis de muestras de voz son proporcionados o generados como de referencia, el proceso de análisis - que es la parte fundamental y esencia del peritaje- no está considerado en esta reflexión, pues es sobre la presentación o redacción de los hallazgos del peritaje que trata este trabajo y no del proceso de recolección de pruebas, hallazgos o resultados de la muestra como tal.

\section{Razonamiento}

En esta sección se realiza el análisis de los resultados obtenidos de cada uno de los elementos que se sometieron a estudio (proporcionados con la voz dubitada como los realizados donde se tiene la voz indubitada), y la descripción de las consideraciones técnico-científicas que dan a los archivos de audio los requisitos mínimos para la identificación y su viabilidad para un análisis biométrico con el fin de determinar la potencial obtención de un resultado falso positivo o falso negativo y más aún si son potencialmente elegibles a ingresar a una base de datos institucional para posteriores cotejos.

A manera de ejemplo, se recomienda este tipo de redacción:

Los archivos de audio 1, 2, 5, 9 y 11, resguardados en formato MP3, no son recomendables para su uso en grabaciones con fines de análisis forense por ser formatos de comprensión con pérdidas, los archivos de video MP4 número 3 y 4 son archivos con audio en formato AAC los cuales no son recomendables para su uso en grabaciones con fines de análisis forense por ser formatos de compresión con pérdidas, los archivos 060768_003 al 060768_011 contenidos en dispositivo de archivo electrónico, comúnmente denominado USB etiquetado con el rótulo manuscrito "llamadas al celular" tienen una utilidad considerada de aceptable; los archivos de sonido contenidos en el CD marca SONY con leyenda escrita de manera impresa "entrevista" y número de identificación G6230KAA70161806 son de utilidad (así la descripción de todos y cada uno de los elementos proporcionados para análisis). 
Se informa que se agrega en sobre bolsa color amarillo, tamaño esquela, dispositivo electrónico comúnmente denominado USB, marca Kingston, color blanco, rotulado con etiqueta impresa la leyenda CI 031/2021-2 PP CI/UIECS/MX/032 Ex17091903 para almacenaje de 21 (veintiuno) archivos de audio elaborados para la práctica pericial, elemento mismo que se integra con su respectiva cadena de custodia.

Momento también en que puede describirse toda la serie de fenómenos o eventos agregados durante la realización del peritaje y todos aquellos comentarios pertinentes y de relevancia para el mismo.

\section{Embalaje}

Documentación y fijación gráfica del material objeto de estudio, donde se muestran las condiciones en que se recibió y como se conservaron durante su manipulación, el grado máximo de integridad en que se recibió y la cadena de custodia. Se recomienda devolver en su empaque y transportador original y en el caso de no ser posible dicha acción referir la causa además de agregar hacer una descripción detallada del nuevo embalaje y material de transportación.

Asimismo, documentar e identificar completamente y de manera amplia y clara cada una de los nuevos elementos generados (archivos de sonido y audio con su respectiva fecha de origen, método de grabación, tamaño, duración y elemento de contención, objeto de transporte y resguardo del mismo, bitácora de entrevistas con personal presuntamente seleccionado para realizar la toma de muestra de voz y mismos a los que se le realizaran los análisis comparativos, consentimientos informados para la exploración y toma de muestras, bitácora del proceso de recolección y generación de muestras que describa la fecha, hora, ubicación y características del lugar donde se realiza el procedimiento, tiempo dedicado, eventos de relevancia, personal presente, material y equipo utilizado; material didáctico generado para la toma de muestras, en casos necesarios en que algún archivo proporcionado haya sufrido algún evento relevante en su integridad describir dicho evento como se resolvió y el material utilizado para la reposición del mismo.

\section{Equipo utilizado}

Se debe especificar el equipo utilizado para la realización del pericial y tener en mente que si existe la posibilidad de replicar o repetir la realización del mismo por cuestionamientos al análisis, esta se debe producir con la máxima similitud de condiciones y de equipo.

- Comúnmente se utiliza una computadora con procesador .... marca... modelo.... Sistema operativo ..... Disco duro de .....

- Monitor adicional marca..... modelo....

- Bafle Marca.... Modelo.... Serie ... para escucha de la reproducción de archivos audibles

- Digitalizador profesional con interfase a computadora a través de puerto USB compatible con entorno Windows

- Micrófono de patrón cardioide, tipo dinámico con frecuencias de respuesta de... impedancia menor de .... Nivel de salida de ...

- Software de análisis de audio (el que se tenga debidamente registrado y de amplio manejo) Voice Passport, Vox Metria, Pro Vox, Computer Speech lab, Adobe, PRATT, etc. 


\section{Conclusión}

Evaluar un nivel de verosimilitud en los informes forenses de voz, implica aplicar una lógica formal, ya que en criminalística las conclusiones pueden estar ligadas a escalas verbales de probabilidad y en ocasiones pueden incluir información subjetiva de base al problema de identificación que se analiza. Por lo tanto, no se pretende asignar probabilidades exactas sino aplicar una metodología eficaz para analizar, criticar y verificar la coherencia de las valoraciones subjetivas que pueden existir ayudándoles a revisar dichas opiniones de forma coherente.

Vale la pena considerar el significado preciso de la diferencia entre carácter objetivo y subjetivo, ya que lo objetivo es considerado como sinónimo de fundado en observaciones medibles, repetibles y confiables; mientras que lo subjetivo es sinónimo de un juicio fundado en conjetura no medible, precipitado, improvisado y difícilmente repetible, aunque su finalidad se centre en el estudio de evaluaciones sobre la probabilidad con la misma profundidad con que se quiere evaluar las probabilidades de naturaleza objetiva, incluso hasta con mayor sentido de responsabilidad.

Así, el informe del "análisis acústico de voz" a los elementos proporcionados y pertenecientes a la carpeta de investigación: 031/2021-2, perteneciente al proceso penal CI/UIECS/ MX/032, expediente 1709-1903, realizado con sistemas automáticos de análisis (oscilográfico, espectrográfico, comparativo de patrones, análisis comparativo de formantes y determinación de frecuencia fundamental, posiciones frecuéntales de los formantes, características espectrales de los formantes y demás técnicas utilizadas), podría indicar que: a) la evidencia dubitada corresponde con la evidencia indubitada con un porcentaje subjetivamente aproximado del $90 \%$ de coincidencia en el cotejo de ambas muestras, b) el hallazgo obtenido al evento de comparación entre muestras dubitadas e indubitadas marca limitada correspondencia del $15 \%$ coincidental a fenómenos acústicos generales, que de acuerdo con el particular y propio saber y entender del perito responsable del estudio, c) los hallazgos obtenidos durante la realización del presente pericial no son concluyentes dado la limitada cantidad y condiciones de almacenaje del material para analizar así como la negativa y falta de cooperación para la toma de muestras de voz indubitada por lo que el presente pericial no es concluyente.

\section{Referencias}

1. Código Penal Federal (México) [Internet] última reforma DOF 12-11-2021; disponible en: http://www.diputados.gob.mx/LeyesBiblio/pdf/CPF.pdf

2. Ley General del Sistema de Seguridad Pública Nacional (México) [Internet] última reforma DOF 20-05-2021. Disponible en: http://www.ordenjuridico.gob.mx/Documentos/ Federal/pdf/wo96855.pdf

3. Constitución Política de los Estados Unidos Mexicanos [Internet].1917. última reforma DOF 28-05-2021 Disponible en: http://www.diputados.gob.mx/LeyesBiblio/pdf_mov/ Constitucion_Politica.pdf

4. Sosa JM. Manual de Criminalística. Limusa 1991.

5. Pérez Bello EA. Ingeniería acústica aplicada a la criminalística "Acústica Forense" [nternet]. [Chile]: Universidad Austral de Chile;2008 Disponible en: http://cybertesis.uach. cl/tesis/uach/2008/bmfcip434i/doc/bmfcip434i.pdf 
6. Molina JJL. La Acústica Forense [Internet].2005 Disponible en: http://portal.uned.es/

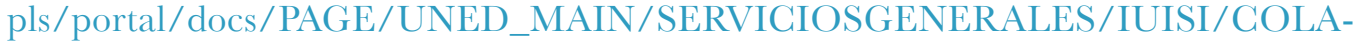
BORACIONES/032\%20DOC037-2005.PDF

7. National Research Council (US). Committee on Evaluation of Sound Spectrograms, et al. On the theory and practice of voice identification. Vol. 4. National Academies, 1979. Doi: https://doi.org/10.17226/19814

8. Morales AQ. Fonética Acústica de la Lengua Española [Internet].Gredos;1981; Disponible en: https://www.researchgate.net/publication/319115428_Antonio_Quilis_Fonetica_ Acustica_de_la_lengua_espanola_Gredos_Madrid_1981_500_pp_BRH_Manuales_49

9. Quaron AQ. Criminalística, Revista Mexicana del Derecho Penal.1961;35-40

10. Gold E, French P. International practices in forensic speaker comparison. International Journal of Speech, Language and the Law. 2011;18(2):293-307. Doi: https://doi. org/10.1558/ijsll.v18i2.293

11. Prieto Castellanos BJ. El uso de los métodos deductivo e inductivo para aumentar la eficiencia del procesamiento de adquisición de evidencias digitales. Cuadernos de contabilidad [nternet].2017;18(46): 18-46. Doi: https://doi.org/10.11144/Javeriana.cc18-46.umdi

12. Rodriguez Bravo A, Lazaro Pernias P, Montoya Villar N, Blanco Pont JM, Suñe DB, Tena Parera D, longhi L, Oliver Comes JM. Identificación perceptiva de locutores para la acústica forense: las RRV. Actas del II congreso de la Sociedad Española de Acústica Forense [Internet]. abril de 2003;23-34. Disponible en: https://www.recercat.cat/handle/2072/449131

13. Bernal Bermudes J, Bobadilla Sánchez J, Gómez Vilda P. Reconocimiento de Voz y Fonética Acústica Alfaomega grupo Editor S.A de C.V. 2000.

14. Cicres J. Comparacion forense de voces mediante el análisis multidimencional de las pausas llenas. Revista Signos estudios de Lingüistica [Internet]. 2014;47(86):365-384. Doi: http://dx.doi.org/10.4067/S0718-09342014000300002

15. Droguett YG. Aplicaciones clinicas del análisis acústico de la voz. Revista Otorrinolaringologia y cirugía de cabeza y cuello [Internet]. 2017;77(4): 474-483 Doi: http://dx.doi. org/10.4067/s0718-48162017000400474 


\section{Anexo. Glosario}

\section{Análisis acústico de la voz}

Es un método de evaluación vocal objetivo y no invasivo utilizado con fines diagnósticos, terapéuticos y de investigación, utilizando registros acústicos obtenidos directamente de la voz del paciente mediante el uso de un micrófono,

\section{Análisis espectográfico}

Es el procedimiento para encontrar la información relativa a los elementos acústicos llamados formantes; dichos formantes son las características fundamentales de la voz: Debiendo precisar que, en todos los espectrogramas ilustrados, el eje horizontal representara al tiempo y el eje vertical a la frecuencia.

\section{Biométrica}

Es el estudio de métodos automáticos para el reconocimiento único de humanos basados en uno o más rasgos conductuales o físicos.

\section{Código HASH}

Algoritmo matemático el cual se utiliza para hacer una comprobación criptográfica o un código de integridad de la evidencia informática o comunicaciones.

\section{Distorsión}

Son alteraciones no deseadas que puede experimentar una señal en su manipulación o transmisión que implican un detrimento en su calidad. Cuando se presentan estas distorsiones en la señal del hablar pueden dar lugar a una nueva componente sonora no deseada. Puede además definirse como la "deformación" que sufre la señal tras su paso por un sistema o al efecto acústico que altera las características espectrales del sonido como adiciones de armónicos no deseados a la señal original.

\section{Editor de audio}

Es una herramienta para la grabación, visualización, selección, reproducción y manipulación de una señal de audio.

\section{Fijación}

Acción o resultado de fijar o fijarse; una foto es una fijación de una imagen en un papel.

\section{Formato MP3 o MPEG-1 layer III}

Se trata de un estándar especialmente diseñado para la codificación y compresión de señales musicales de audio. Cuando se quiere codificar audio con tasas binarias pequeñas es preciso recurrir a codificaciones con pérdidas en la señal, por lo que la información eliminada es irrecuperable en la descodificación.

\section{Formato MP4 o MPEG-4}

Contiene video codificado con MPEG-4 y audio codificado con Advanced Audio Coding (AAC) bajo la extensión .mp4. El formato AAC aplica una forma de compresión que reduce alguno de los datos de audio y que se denomina "compresión con pérdidas" esto quiere decir que se eliminan algunos de los datos de audio, por ejemplo, las frecuencias inaudibles para los seres humanos, de esta manera que se pueda obtener un mayor grado de reducción del tamaño del archivo, aunque este archivo resultante suena igual al original en la práctica. 


\section{Formato WAV}

Es un formato de audio sin perdida (PCM lineal) que facilita la codificación de sonido.

\section{Frecuencia}

Magnitud que determina el número de repeticiones de un fenómeno físico por unidad de tiempo.

\section{Ininteligible}

Imposibilidad de ser comprendido o interpretado.

\section{Identificación por la voz}

Técnica para identificar personas a partir de las características de su voz.

\section{Modelo}

Cuando nos referimos a modelos dentro de un sistema nos referimos a modelos de locutor realizados a partir de uno o varios archivos de audio. Se genera un modelo por locutor, tanto si éste es un individuo identificado como si es una reseña (audio) no identificada.

\section{Oscilograma}

Representación de la amplitud de la señal vocal expresada en pascales (eje vertical) en función del tiempo expresado en segundos (eje horizontal).

\section{Requisitos científicos-técnicos}

Descripción de los requisitos mínimos de un conjunto de evidencias para la identificación en la base de datos institucional y en su caso viables para un análisis biométrico.

\section{Reverberación}

Es el efecto natural que se produce en un espacio cerrado cuando el sonido rebota en las paredes, techo y suelo hasta formar un conglomerado de estos, los cuales están tan cercanos entre si que se hace imposible percibirlos por separado, por lo que percibimos un solo sonido constante parecido al sonido original que originó el efecto.

\section{Ruido}

Cualquier señal no deseada que interfiere la transmisión, recepción, percepción o interpretación de una señal útil (sonido de interés). El ruido puede ser definido como las componentes no deseadas de una señal, ejemplos de ruido en una señal de habla pudieran ser ruido de la red, música de fondo, reverberaciones e incluso otras señales vocales.

\section{Saturación}

Ocurre cuando el nivel de la señal entrante excede los límites de la representación codificada de la misma, en cualquier momento de la grabación y procesamiento de la señal.

\section{Solapamiento}

Es un concepto descriptivo que se aplica a los casos de habla simultanea (overlap) entre dos o más locutores.

\section{Valoración subjetiva}

Es el procedimiento de escuchar los audios del material problema en repetidas ocasiones por el experto, tantas cuantas sean necesarias, para poder apreciar la cantidad de locutores y ser considerados como voz o voces de interés valorándose solo de puramente forma auditiva; con 
volumen suficiente para ser perceptible al oído humano de quien los escucha, refiriéndose además la presencia de ruido de fondo, solapamientos entre voces, distorsión, saturación, reverberación, etc. En este proceso se realiza una primera selección de los audios, descartando o aceptando los que se consideren válidos para una siguiente etapa de estudio.

\section{Volumen de disco}

En sistemas operativos, el termino volumen hace referencia a una única área accesible de almacenamiento, como ser una partición de un disco duro, el volumen posee una letra de unidad (C, D, E, etc.) un tipo de sistema de archivo y un medio de almacenamiento correspondiente (disco duro, CD-ROM, Disquetera, etc.

\section{Voz dubitada}

Voz grabada de la que existen dudas en torno a la identidad de la persona que habla.

\section{Voz indubitada}

Grabación de la que no existen dudas respecto a la identificación de la persona que habla. 